\title{
Computer-assisted quantitative evaluation of bisphosphonate treatment for Paget's disease of bone using the bone scan index
}

\author{
SATOSHI NAGANO $^{1 *}$, SHUNSUKE NAKAMURA ${ }^{1 *}$, HIROFUMI SHIMADA $^{1}$, MASAHIRO YOKOUCHI $^{1}$, \\ TAKAO SETOGUCHI $^{2}$, YASUHIRO ISHIDOU ${ }^{3}$, HIROMI SASAKI ${ }^{1}$ and SETSURO KOMIYA ${ }^{1}$ \\ ${ }^{1}$ Department of Orthopaedic Surgery; ${ }^{2}$ The Near-Future Locomotor Organ Medicine Creation Course (Kusunoki Kai); \\ ${ }^{3}$ Department of Medical Joint Materials, Graduate School of Medical and Dental Sciences, \\ Kagoshima University, Kagoshima 890-8520, Japan
}

Received August 13, 2015; Accepted September 27, 2016

DOI: $10.3892 / \mathrm{etm} .2016 .3899$

\begin{abstract}
The purpose of the present study was to analyze the effect of treatment of Paget's disease of bone (PDB) with bone scintigraphy using a computer-assisted diagnosis system (BONENAVI) that quantitatively evaluates bone metabolism. Seven patients with PDB (three male, four female; average age, 60 years; age range, 33-80 years) underwent bone scintigraphy and measurement of serum alkaline phosphatase (ALP), bone-specific ALP (BAP), serum cross-linked N-telopeptide (NTx) of type I collagen, urinary NTx, and deoxypyridinoline (DPD) before and after bisphosphonate treatment. Bone scan index (BSI), artificial neural network (ANN) value, and hotspot number (HSn) were calculated using BONENAVI software. Mean follow-up period was 22 months (range, 11-35 months). Among three BONENAVI parameters (ANN, BSI, and HSn), only BSI was significantly lower after bisphosphonate treatment as compared with before. All bone metabolic markers excluding DPD were significantly lower following bisphosphonate treatment than before. Bone formation markers (ALP and BAP) were significantly lower than bone resorption markers (U-NTx and S-NTx). The correlation of BONENAVI parameters with four bone metabolic markers was analyzed before and after bisphosphonate treatment. Before treatment, the majority of the four markers did not correlate with the BONENAVI parameters. In contrast, post-treatment ALP, BAP, and U-NTx were significantly correlated with BSI and HSn. To the best of our knowledge, this is the first study to evaluate the treatment of
\end{abstract}

Correspondence to: Dr Satoshi Nagano, Department of Orthopaedic Surgery, Graduate School of Medical and Dental Sciences, Kagoshima University, 8-35-1 Sakuragaoka, Kagoshima, Kagoshima 890-8520, Japan

E-mail: naga@m2.kufm.kagoshima-u.ac.jp

*Contributed equally

Key words: Paget's disease of bone, bone scintigraphy, computer-assisted analytical system, bone metabolic marker, bisphosphonate
PDB by bone scintigraphy using a computer-assisted diagnosis system that quantitatively evaluates bone metabolism. The findings demonstrated that, using BONENAVI software, bone scintigraphy is able to quantitatively and spatially evaluate the bisphosphonate treatment effect, particularly in patients with polyostotic PDB.

\section{Introduction}

Paget's disease of bone (PDB) is a metabolic bone disease characterized by increased bone resorption and active bone formation. The prevalence of PDB in Japan is relatively lower $(2.8 / 1,000,000$ individuals) than that of North American and European countries (1). Mixed lytic and sclerotic changes are present on the roentgenograms of patients with PDB, and bone scintigraphy using technetium $-99^{\mathrm{m}}$ is often more sensitive than plain radiographs (2). Because of the lower prevalence of PDB observed in Japan than in Western countries, diagnosis of PDB remains difficult for Japanese physicians. In a report by the committee for PDB within the Japan Osteoporosis Society, 55\% of Japanese patients with PDB underwent diagnostic biopsy of the affected bones (1). Since publication of the report, PDB has become more widely recognized and diagnosis without biopsy has become more common. Another characteristic sign of PDB is elevation of serum alkaline phosphatase (ALP) levels. Generally, diagnosis of PDB is confirmed by radiological findings, high tracer accumulation in bone scintigraphy, and a high serum ALP level $(1,2)$. Although PDB is often asymptomatic, it can cause bone pain, osteoarthritis, pathological fractures, bone deformity, deafness, and nerve compression syndromes (3). Patients with symptomatic PDB or PDB that affects the weight-bearing bones are candidates for medical treatments to reduce bone turnover. The treatment of choice is a potent nitrogen-containing bisphosphonate, such as alendronate, risedronate, pamidronate or zoledronic acid. Treatment monitoring is divided into two categories, biochemical marker analysis and imaging analysis. The total serum ALP level is the most commonly used marker due to its low cost and high reproducibility (2). We propose that bone scintigraphy should be the imaging technique of choice, as tracer uptake is directly associated with disease activity and may be observed in advance of any radiographic evidence (4). For quantitative bone scintigraphic analysis; however, obtaining 
Table I. Patient characteristics.

\begin{tabular}{|c|c|c|c|c|c|c|}
\hline Number & Age & Gender & Affected site & Biopsy & Symptom & Bisphosphonate \\
\hline 1 & 59 & Female & Skull, pelvis, femur & + & & $\mathrm{E}, \mathrm{R}$ \\
\hline 2 & 76 & Female & Humerus & + & + & \\
\hline 3 & 48 & Male & Cervical spine, humerus & + & & $\mathrm{R}^{\mathrm{a}}$ \\
\hline 4 & 80 & Male & Pelvis, humerus & & + & $\mathrm{R}$ \\
\hline 5 & 33 & Female & Skull, femur & & & $\mathrm{R}^{\mathrm{a}}$ \\
\hline 6 & 64 & Female & Pelvis & & & $M^{\mathrm{a}}$ \\
\hline 7 & 59 & Male & Sacrum & & + & $\mathrm{R}$ \\
\hline
\end{tabular}

${ }^{\mathrm{a}}$ Reduced dose. $\mathrm{E}$, etidronate; $\mathrm{R}$, risedronate; $\mathrm{M}$, minodronate.

results at different time points is not straightforward and may be inaccurate due to the lack of suitable image analysis software. Computer-assisted diagnosis (CAD) systems for bone scintigraphy, which predominantly target metastatic bone tumors, have recently been developed $(5,6)$. The representative system, BONENAVI (Fujifilm RI Pharma, Tokyo, Japan), is based on artificial neural networks (ANNs) and evaluates the risk of bone metastasis from bone scintigraphy data. BONENAVI is a bone scintigraphy diagnosis support software established with a Japanese database. It is used for the diagnosis and follow-up observation of patients with bone metastasis $(7,8)$. This system evaluates three parameters: Bone scan index (BSI), ANN value, and hotspot number (HSn). The ANN value ranges from 0 to 1 and indicates the probability of metastasis as follows: $\mathrm{ANN}=0.00-0.25$, bone metastasis is not suspected in the bone scans; $\mathrm{ANN}=0.26-0.50$, bone metastasis may not be fully excluded; $\mathrm{ANN}=0.51-0.75$, bone metastasis is suspected; and ANN=0.76-1.00, bone metastasis is highly suspected (9). BSI represents the total amount of abnormal tracer accumulation and is shown as a value relative to the whole body (0-100\%). HSn is the number of abnormal lesions and is determined by the ANN. We hypothesized that the BSI and HSn may be useful for the quantitative evaluation of bone metabolism in patients with PDB. Furthermore, whether BONENAVI parameters correlate with well-known bone metabolic markers was evaluated.

\section{Materials and methods}

Patients. The present study included seven Japanese patients with PDB diagnosed in our department who were treated between 2008 and 2011. The seven patients comprised four female and three male patients with an average age of 60 years (range, 33-80 years). The characteristics of the patients are summarized in Table I. Three patients underwent biopsy to establish the diagnosis of PDB. In accordance with the guidelines of the Japanese Committee for PDB within the Japan Osteoporosis Society (10), we have recently begun to establish the diagnosis of PDB based on imaging studies and serum bone metabolic markers, and this was performed in Patients 4-7 (Table I). All patients underwent bisphosphonate treatment under informed consent. Three patients exhibited symptoms associated with the affected bone. The remaining four patients were treated owing to concern regarding future complications associated with the affected weight-bearing bones (Patients 1,5 and 6) or cervical spine (Patient 3). Four patients received $17.5 \mathrm{mg}$ risedronate (Eisai, Co., Ltd., Tokyo, Japan) daily for 8 weeks in accordance with previously established Japanese guidelines (10). The remaining three patients were administered a reduced dose of weekly $17.5 \mathrm{mg}$ risedronate or monthly minodronate (Ono Pharmaceutical Co., Ltd., Osaka, Japan) due to of adverse effects (gastritis in Patient 2) or lack of symptoms (Patients 5 and 6).

Bone metabolic markers. Serum total ALP, bone-specific ALP (BAP), and type I collagen cross-linked N-telopeptide (sNTx) levels were examined before and after the initiation of therapy. Urinary markers, deoxypyridinoline (DPD) and urine NTx (uNTx), were also analyzed. ALP was analyzed via a colorimetric assay using p-nitrophenyl phosphate substrate according to the manufacturer's instructions (Iatro ALP; LSI Medience Co., Tokyo, Japan). BAP/DPD and NTx (serum and urine) were analyzed by enzyme immunoassay and enzyme-linked immunosorbent assay, respectively, at a clinical laboratory (SRL Inc., Kagoshima, Japan). Both DPD and uNTx were adjusted by the serum creatinine level to account for differences in urine volume.

Bone scintigraphy and BONENAVI analysis. Whole-body bone scintigraphy images were acquired $4 \mathrm{~h}$ after intravenous injection of $740 \mathrm{MBq}$ technetium-99 ${ }^{\mathrm{m}}$-methylene diphosphonate (Fujifilm RI Pharma) using a dual-head gamma camera equipped with low-to-mid-energy general-purpose collimators (Symbia T6; Siemens Medical Solutions, Malvern, PA, USA).

BONENAVI 2.0 (Fujifilm RI Pharma and EXINI Bone; EXINI Diagnostics, Lund, Sweden) was used to calculate the BSI, ANN, and HSn. Region-based ANNs in the range of 0.00 to 0.50 were expressed as blue spots, whereas those ranging from 0.51 to 1.00 were seen as red hotspots (Fig. 1). Based on the number of red areas, the software calculates the ANN to estimate the risk of bone metastasis $(11,12)$. BSI and HSn were monitored as an objective evaluation of the medical treatment (Fig. 1).

Statistical analysis. Alterations in the values of the bone metabolic markers or BONENAVI parameters before and after bisphosphonate treatment were analyzed by Student's t-test. Correlations between BONENAVI parameters (ANN, BSI, and HSn) and bone metabolism markers (ALP, BAP, sNTx, and $\mathrm{uNTx}$ ) were analyzed by Pearson's correlation coefficient. 
Table II. Pearson's correlation index between BONENAVI system parameters and bone metabolic markers.

\begin{tabular}{lccccccc}
\hline & \multicolumn{3}{c}{ Pre-treatment } & & \multicolumn{3}{c}{ Post-treatment } \\
\cline { 2 - 3 } \cline { 8 - 9 } & ANN & BSI & HSn & & ANN & BSI & HSn \\
\hline ALP & 0.24 & 0.16 & 0.53 & & 0.25 & $0.96^{\mathrm{a}}$ & $0.96^{\mathrm{a}}$ \\
BAP & 0.29 & 0.35 & 0.75 & & 0.33 & $0.94^{\mathrm{a}}$ & $0.96^{\mathrm{a}}$ \\
sNTx & 0.63 & 0.28 & 0.74 & & 0.30 & 0.73 & 0.75 \\
uNTx & 0.59 & 0.46 & $0.88^{\mathrm{a}}$ & & 0.45 & $0.98^{\mathrm{a}}$ & $0.96^{\mathrm{a}}$ \\
\hline
\end{tabular}

${ }^{\mathrm{a}} \mathrm{P}<0.05$. BSI, bone scan index; $\mathrm{ANN}$, artificial neural network value; HSn, hotspot number.

$\mathrm{P}<0.05$ was considered to indicate a statistically significant difference.

Ethics statement. All procedures involving human participants were performed in accordance with the 1964 Helsinki declaration and its later amendments or comparable ethical standards. Informed consent was obtained from all individual participants included in the study.

\section{Results}

Bisphosphonate treatment reduces all three BONENAVI parameters (BSI, ANN and HSn). A 64-year-old female (patient 5) exhibited a high pretreatment ANN (0.85) prior to bisphosphonate treatment (Fig. 1A). Post-treatment BONENAVI analysis showed a reduction in all three parameters, namely BSI, ANN and HSn (Fig. 1B). Notably, red hotspots were located around the right pelvis.

Bisphosphonate treatment significantly reduces $A L P, B A P$, $s N T x$ and $u N T x$ levels. The average serum ALP level was significantly lower following bisphosphonate treatment, compared with before ( $\mathrm{P}=0.0052$; Fig. 2A). Serum levels of the other bone formation marker, BAP, were also significantly lower after treatment $(48.0 \pm 19.0)$ as compared with pre-treatment $(93.8 \pm 23.0$; $\mathrm{P}=0.003$; Fig. 2B). Among all bone resorption markers, the reductions of sNTx and uNTx were statistically significant ( $\mathrm{P}=0.01$ and $\mathrm{P}=0.04$, respectively). DPD was the only marker to not exhibit a significant reduction following bisphosphonate treatment.

Bisphosphonate treatment significantly reduces BSI, as detected by BONENAVI. Among the three BONENAVI parameters, only BSI significantly decreased from a pretreatment value of $3.33 \pm 0.90$ to a post-treatment value of $1.84 \pm 1.00$ (Fig. 3A). Neither the ANN nor the HSn significantly changed with treatment (Fig. 3B and C, respectively).

Bone formation and reabsorption markers are significantly correlated. Correlation analysis between the BONENAVI parameters and bone metabolism markers revealed almost no correlation with the pretreatment values (Table II). On the other hand, the post-treatment BSI and HSn showed a significant
A

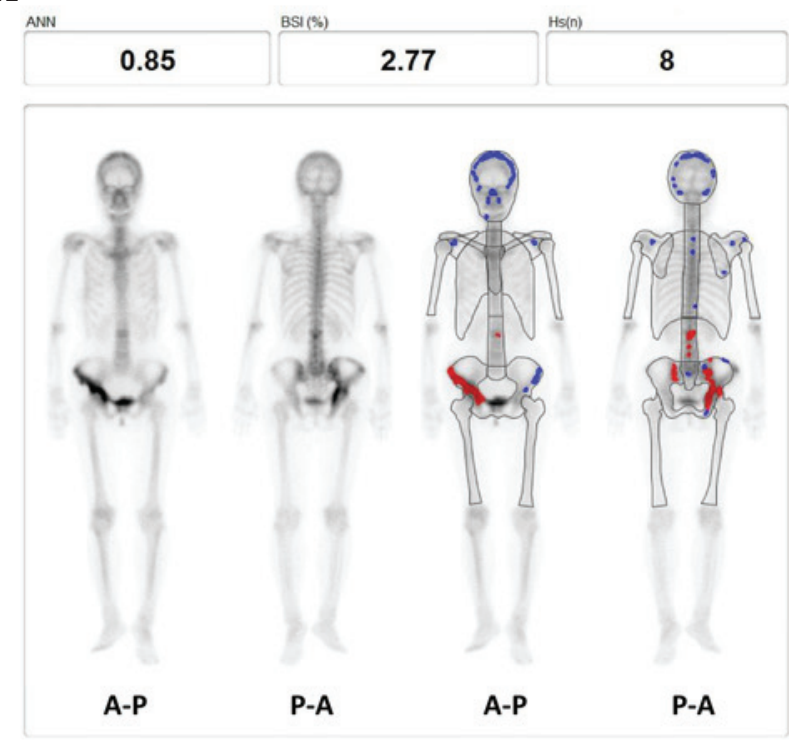

B

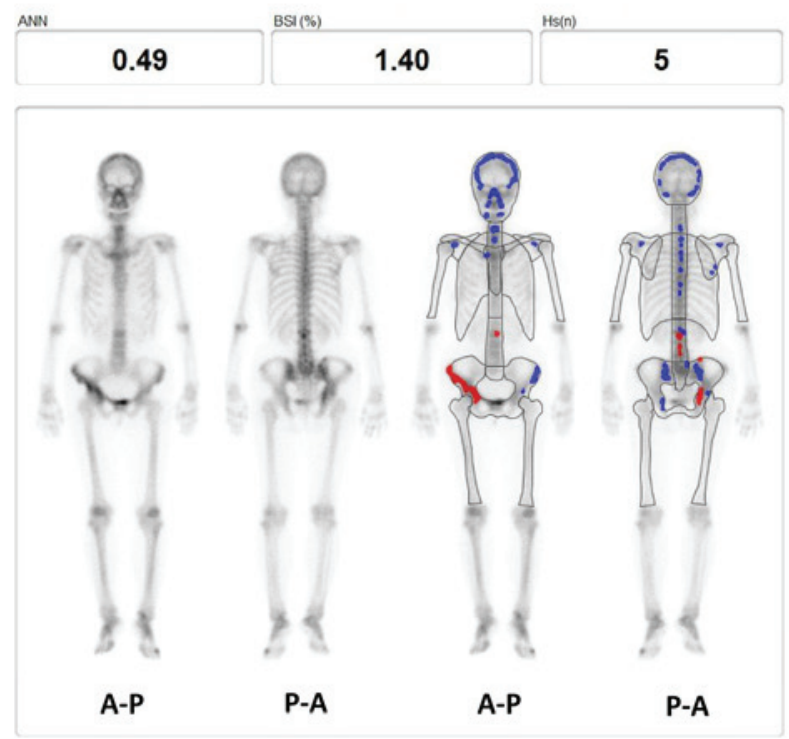

Figure 1. BONENAVI analysis results. (A) A 64-year-old woman (patient 5) exhibited a high pretreatment ANN (0.85) prior to treatment. (B) Post-treatment BONENAVI analysis showed a reduction in all three parameters. Region-based ANNs in the range of 0.00 to 0.50 were expressed as blue spots, whereas those ranging from 0.51 to 1.00 were expressed as red hotspots. Notably, red hotspots were located around the right pelvis. ANN, artificial neural network value.

correlation with the bone formation and resorption markers (ALP, BAP, and uNTx) (Fig. 4).

Representative case. A representative case is shown in Fig. 5. This 59-year-old man had been referred to our institution due to abnormal tracer accumulation in the sacrum during a bone scan, accompanies by elevated ALP levels (Fig. 5A-D). Positron-emission tomography with fluorodeoxyglucose was performed, which revealed no accumulation of fluorodeoxyglucose in the sacral area (Fig. 5E). The patient was diagnosed with PDB and administered risedronate treatment for 8 weeks. As compared with prior to treatment (Fig. 5F), post-treatment BONENAVI imaging revealed a markedly 
A

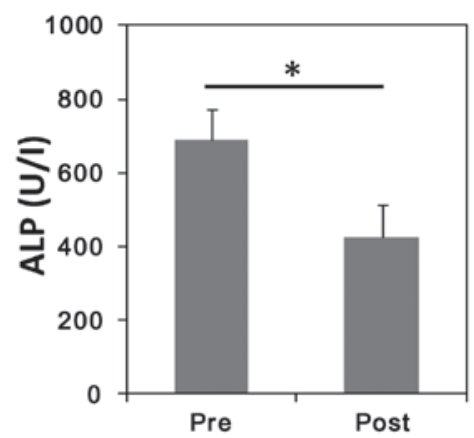

C

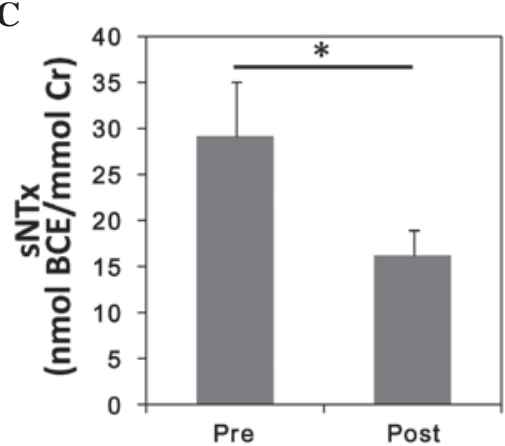

B

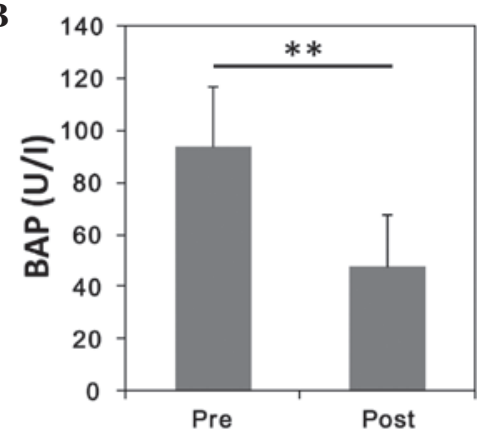

D

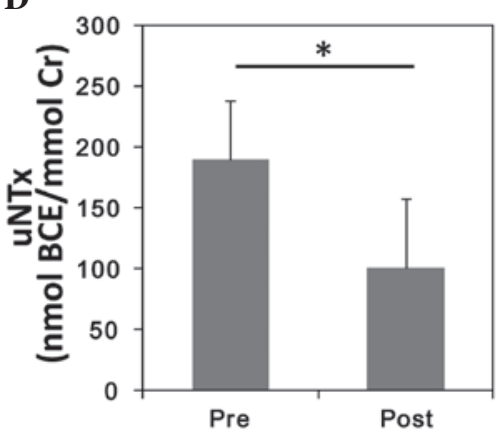

$\mathbf{E}$

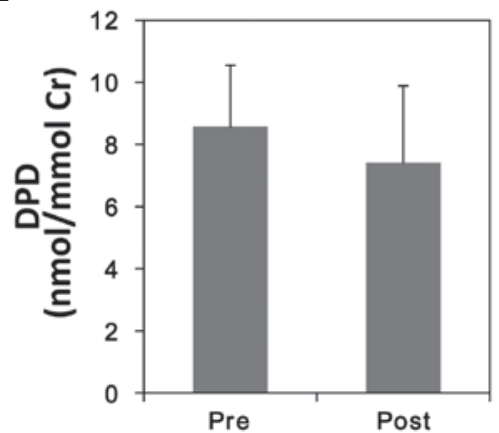

Figure 2. Alterations in bone metabolic marker levels before and after treatment with bisphosphonate. Mean values of (A) ALP, (B) BAP, (C) sNTx, (D) uNTx, and (E) urinary DPD. "P<0.05; * $\mathrm{P}<0.005$. ALP, serum alkaline phosphatase; BAP, serum bone-specific ALP; BCE, bone collagen equivalent; sNTx, serum type I collagen cross-linked N-telopeptide; uNTx, urinary NTx; DPD, deoxypyridinoline; Cr, creatinine.

A

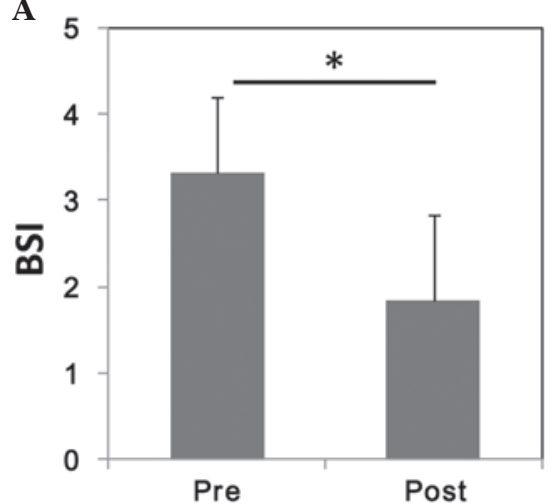

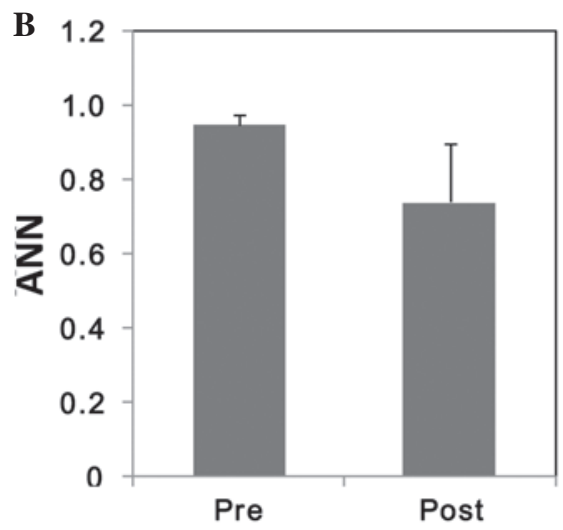

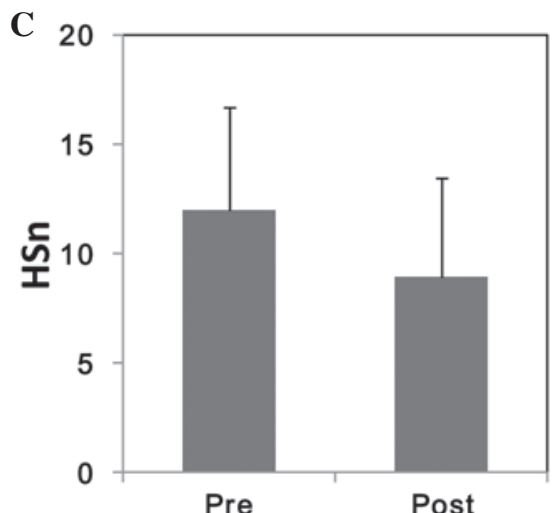

Figure 3. Parameters of BONENAVI analysis. Pre and post treatment values of (A) BSI, (B) ANN.and (C) HSn. Only the BSI was significantly reduced by bisphosphonate treatment. "P<0.05. BSI, bone scan index; ANN, artificial neural network; Hsn, number of hotspots.

reduced tracer-positive area on the bone scan (Fig. 5G). The ALP level also significantly decreased from a pretreatment value of $498 \mathrm{U} / 1$ to a post-treatment value of $269 \mathrm{U} / 1$.

\section{Discussion}

The concept of the BSI for quantitative evaluation of bone 
A

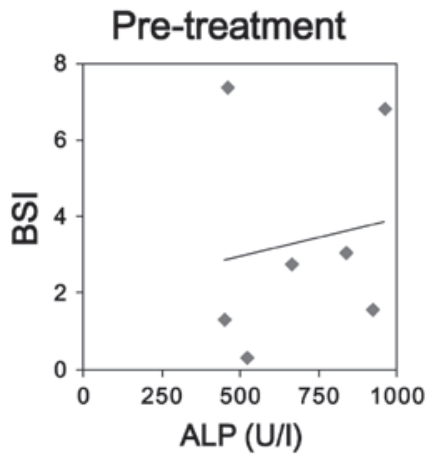

B

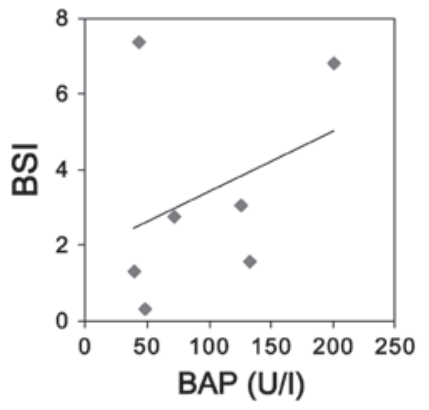

C

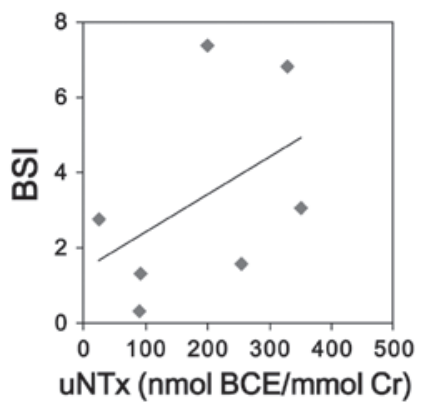

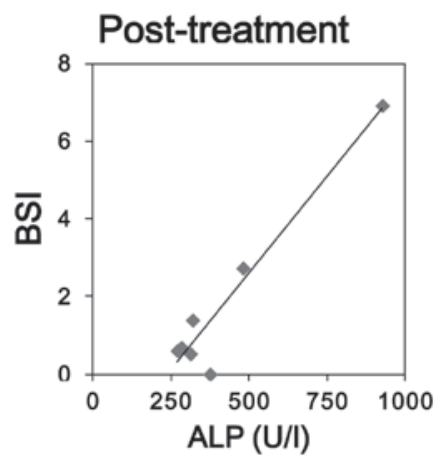
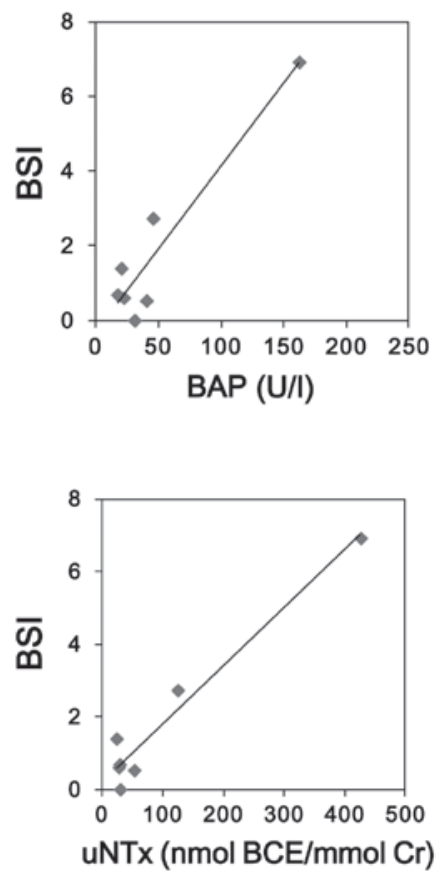

Figure 4. Scatter plot diagrams between (A) ALP, (B) BAP and (C) UNTx bone metabolic markers and BSI. Notably, post-treatment exhibited a good correlation between the two values. ALP, alkaline phosphatase; BAP, bone-specific ALP; NTx, serum cross-linked N-telopeptide; BSI, bone scan index.

scans in patients with cancer was first established by a group from the Memorial Sloan-Kettering Cancer Center (13). They reported that the BSI may be useful for stratifying patients with prostate cancer entering treatment protocols. However, the BSI was calculated from the weight and the fractional involvement of each bone, expressed as percentages of the entire skeleton; this is largely dependent upon each observer's visual evaluation (13). Following establishment of the CAD-based BONENAVI system for automatic calculation of the BSI, its usefulness in the diagnosis and monitoring of bone metastasis has been reported by several authors $(11,14,15)$. BONENAVI is now routinely used during bone scans in various facilities, including our institution. Therefore, data from patients with bone metastasis will be accumulated to determine how to adapt BONENAVI analysis in the management of bone metastases in the future. To our knowledge, no studies have evaluated the use of BONENAVI in patients with PDB. Although the number of patients in the present study was limited by the extremely low incidence of PDB in Japan, the present findings demonstrated that the BSI reflects the response to bisphosphonate treatment in patients with $\mathrm{PDB}$. The advantage of using BONENAVI over the measurement of bone metabolic markers is the ability to spatially evaluate bone metabolism. It would be helpful to evaluate the correlation between changes in bone metabolism and symptoms associated with the affected bone after bisphosphonate treatment in patients with polyostotic PDB. However, it is not possible to separately evaluate each affected lesion using only bone metabolic markers, particularly in patients with polyostotic PDB. For instance, Patient 4 had mild pain in the right upper extremity. Pretreatment analysis revealed hotspots predominantly in the right humerus and left pelvis (Table I). Although the hotspots in the pelvic area remained unchanged after treatment, accumulation in the left humerus was reduced (blue), resulting in relief of the patient's pain. Therefore, we consider that the bisphosphonate treatment in this patient was effective for the symptomatic lesion in the humerus, but not substantially for the pelvic lesion.

Biochemical assessment of PDB has been studied previously (16). Since bisphosphonates are antiresorptive, three markers in this study were monitored in the present study (sNTx, uNTx, and DPD). The present finding that uNTx better demonstrated the effect of bisphosphonate treatment than DPD is consistent with previous reports $(16,17)$. It has 
A

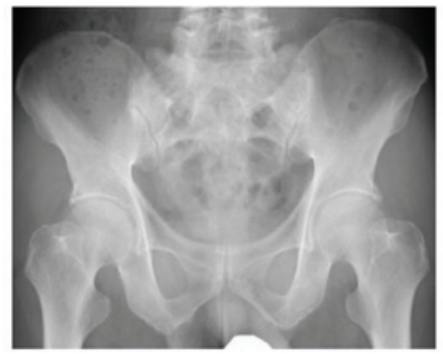

D

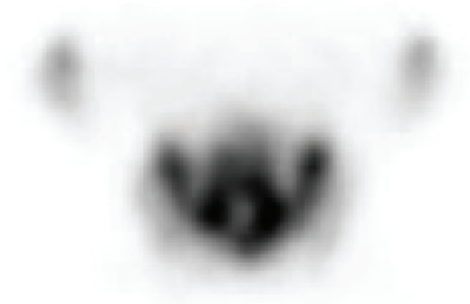

F

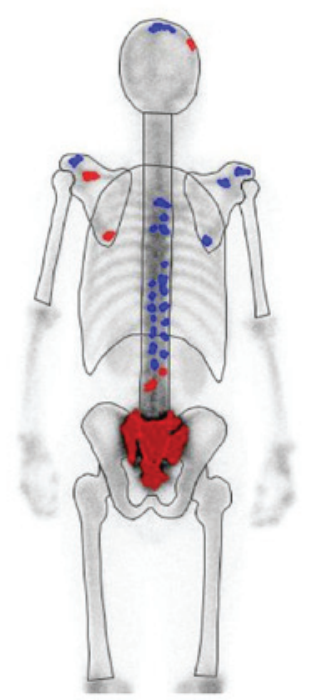

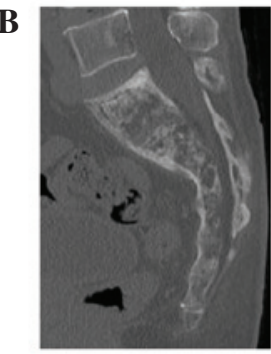

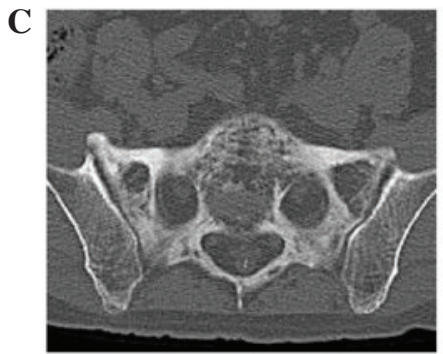

E

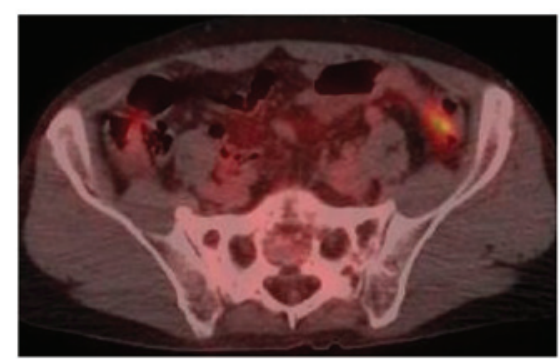

G

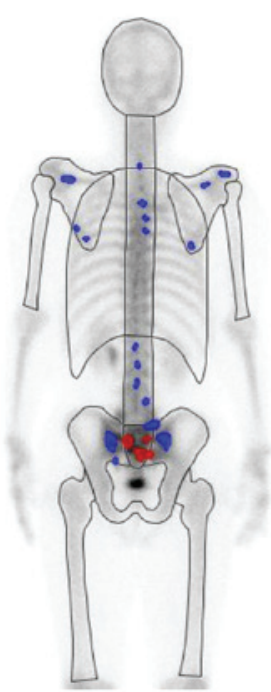

Figure 5. A representive case of a 59-year-old man (Patient 7) with PDB in the sacrum. (A) Plain radiograph showed sclerotic alterations in the sacrum. (B and C) Computed tomography demonstrated a thickened cortex and irregular sclerosis of the cancellous bone. (D) An axial-view bone scan exhibited marked tracer accumulation in the sacrum. (E) Positron-emission tomography with fluorodeoxyglucose revealed no accumulation in the sacrum. The patient was diagnosed with PDB and underwent treatment with risedronate. (F) Pretreatment BONENAVI analysis (posterior view) demonstrated hotspots predominantly around the sacrum. (G) Following treatment with risedronate, a marked reduction in the size of the hotspot area was observed. PDB, Paget's disease of bone.

previously been demonstrated that short-term reduction of uNTx may predict the final post-treatment result evaluated by ALP (18). As markers of bone formation, ALP, BAP, and procollagen type $1 \mathrm{~N}$-terminal propeptide perform similarly in the diagnosis and assessment of polyostotic PDB (17). In particular, in patients with monostotic PDB with limited disease activity, BAP has been shown to be increased despite a normal ALP level (19). In the present patient series, both BAP and ALP were elevated in all patients and were demonstrated to be significantly reduced after treatment, as compared with before. For the reasons outlined in the present study, in addition to previous research and its cost-effectiveness, we conclude that ALP has the most established role in the evaluation and monitoring of PDB $(16,17)$.

It should be emphasized that the present study is the first to have shown a good correlation between quantitative bone scan results and biomarkers in patients with PDB. A previous study evaluated bone scan results using a manually and visually quantified 'scintigraphic index' (16). However, to become a useful tool for physicians, such analysis should not only provide reproducible results, but its performance should also feasible. BONENAVI is a reliable analysis of bone metabolic diseases, including PDB; however, how it is correlated with bone metabolic markers remains unknown. In this context, the correlation index between BONENAVI parameters and biochemical markers was analyzed. Unexpectedly, the pretreatment BONENAVI values were not significantly correlated with any bone metabolic markers, except HSn and UNTx (Table II). Iwase et al (20) recently studied the correlation between the BSI and bone metabolic markers in patients with bone metastatic breast cancer. They showed that BSI was significantly correlated with the ALP level at 
the time of initial treatment. The present study demonstrated that the post-treatment values of ALP, BAP, and uNTx were significantly correlated with the BSI and HSn (Table II). Therefore, although the BSI is not correlated with pretreatment biochemical markers, it is an imaging biomarker that is as reliable as bone metabolic markers to evaluate the efficacy of bisphosphonate treatment.

The main limitation of the present study was the small sample size, which was largely due to the low prevalence rate of PDB in Japan (1). Based on this pilot study, further validation studies from Western countries are required to elucidate whether this CAD-based system is useful in the management of PDB.

In conclusion, the present study is the first to demonstrate that automated quantitative and BONENAVI qualitative evaluation software for bone scans may be used to monitor treatment response in patients with PDB. Although the majority of the bone metabolic markers decreased in response to bisphosphonate treatment, only BSI significantly decreased among the three BONENAVI parameters evaluated. A significant correlation between the post-treatment BSI and bone metabolic markers was also demonstrated. The use of BONENAVI software in combination with bone metabolic markers may allow bone scintigraphy to quantitatively and spatially evaluate treatment responses to bisphosphonates, particularly in patients with polyostotic PDB.

\section{References}

1. Hashimoto J, Ohno I, Nakatsuka K, Yoshimura N, Takata S, Zamma M, Yabe H, Abe S, Terada M, Yoh K, et al: Prevalence and clinical features of Paget's disease of bone in Japan. J Bone Miner Metab 24: 186-190, 2006.

2. Langston AL and Ralston SH: Management of Paget's disease of bone. Rheumatology (Oxford) 43: 955-959, 2004.

3. Ralston SH, Langston AL and Reid IR: Pathogenesis and management of Paget's disease of bone. Lancet 372: 155-163, 2008 .

4. Scutellari PN, Giorgi A, De Sario V and Campanati P: Correlation of multimodality imaging in Paget's disease of bone. Radiol Med 110: 603-615, 2005 (In English, Italian).

5. Sadik M, Jakobsson D, Olofsson F, Ohlsson M, Suurkula M and Edenbrandt L: A new computer-based decision-support system for the interpretation of bone scans. Nucl Med Commun 27: 417-423, 2006

6. Sadik M, Hamadeh I, Nordblom P, Suurkula M, Höglund P, Ohlsson $\mathrm{M}$ and Edenbrandt L: Computer-assisted interpretation of planar whole-body bone scans. J Nucl Med 49: 1958-1965, 2008.
7. Wakabayashi H, Nakajima K, Mizokami A, Namiki M, Inaki A, Taki J and Kinuya S: Bone scintigraphy as a new imaging biomarker: The relationship between bone scan index and bone metabolic markers in prostate cancer patients with bone metastases. Ann Nucl Med 27: 802-807, 2013.

8. Koizumi M, Wagatsuma K, Miyaji N, Murata T, Miwa K, Takiguchi T, Makino T and Koyama M: Evaluation of a computer-assisted diagnosis system, BONENAVI version 2, for bone scintigraphy in cancer patients in a routine clinical setting. Ann Nucl Med 29: 138-148, 2015

9. Horikoshi H, Kikuchi A, Onoguchi M, Sjöstrand K and Edenbrandt L: Computer-aided diagnosis system for bone scintigrams from Japanese patients: Importance of training database. Ann Nucl Med 26: 622-626, 2012.

10. Takata S, Hashimoto J, Nakatsuka K, Yoshimura N, Yoh K, Ohno I, Yabe H, Abe S, Fukunaga M, Terada M, et al: Guidelines for diagnosis and management of Paget's disease of bone in Japan. J Bone Miner Metab 24: 359-367, 2006.

11. Koizumi M, Wagatsuma K, Miyaji N, Murata T, Miwa K, Takiguchi T, Makino $\mathrm{T}$ and Koyama M: Evaluation of a computer-assisted diagnosis system, BONENAVI version 2, for bone scintigraphy in cancer patients in a routine clinical setting. Ann Nucl Med 29: 138-148, 2015.

12. Kikushima S, Hanawa N and Kotake F: Diagnostic performance of bone scintigraphy analyzed by three artificial neural network systems. Ann Nucl Med 29: 125-131, 2015.

13. Imbriaco M, Larson SM, Yeung HW, Mawlawi OR, Erdi Y, Venkatraman ES and Scher HI: A new parameter for measuring metastatic bone involvement by prostate cancer: The bone scan index. Clin Cancer Res 4: 1765-1772, 1998.

14. Tokuda O, Harada Y, Ohishi Y, Matsunaga N and Edenbrandt L: Investigation of computer-aided diagnosis system for bone scans: A retrospective analysis in 406 patients. Ann Nucl Med 28: 329-339, 2014.

15. Takahashi Y, Yoshimura M, Suzuki K, Hashimoto T, Hirose H, Uchida K, Inoue S, Koizumi K and Tokuuye K: Assessment of bone scans in advanced prostate carcinoma using fully automated and semi-automated bone scan index methods. Ann Nucl Med 26: 586-593, 2012.

16. Reid IR, Davidson JS, Wattie D, Wu F, Lucas J, Gamble GD, Rutland MD and Cundy T: Comparative responses of bone turnover markers to bisphosphonate therapy in Paget's disease of bone. Bone 35: 224-230, 2004.

17. Shankar S and Hosking DJ: Biochemical assessment of Paget's disease of bone. J Bone Miner Res 21 (Suppl 2): P22-P27, 2006.

18. Papapoulos SE and Frölich M: Prediction of the outcome of treatment of Paget's disease of bone with bisphosphonates from short-term changes in the rate of bone resorption. J Clin Endocrinol Metab 81: 3993-3997, 1996.

19. Alvarez L, Guañabens N, Peris P, Monegal A, Bedini JL, Deulofeu R, Martinez de Osaba MJ, Muñoz-Gomez J, Rivera-Fillat F and Ballesta AM: Discriminative value of biochemical markers of bone turnover in assessing the activity of Paget's disease. J Bone Miner Res 10: 458-465, 1995.

20. Iwase $T$, Yamamoto $N$, Ichihara $H$, Togawa $T$, Nagashima $T$ and Miyazaki M: The relationship between skeletal-related events and bone scan index for the treatment of bone metastasis with breast cancer patients. Medicine (Baltimore) 93: e269, 2014. 